\title{
Electron energy spectrum in core-shell elliptic quantum wire
}

\author{
V.Holovatsky, V.Gutsul \\ Fedkovych Chernivtsi National University, 2, Kotsyubinskoho St., Chernivtsi, 58012, Ukraine*
}

Received September 4, 2006, in final form January 4, 2007

\begin{abstract}
The electron energy spectrum in core-shell elliptic quantum wire and elliptic semiconductor nanotubes are investigated within the effective mass approximation. The solution of Schrodinger equation based on the Mathieu functions is obtained in elliptic coordinates. The dependences of the electron size quantization spectrum on the size and shape of the core-shell nanowire and nanotube are calculated. It is shown that the ellipticity of a quantum wire leads to the break of degeneration of quasiparticle energy spectrum. The dependences of the energy of odd and even electron states on the ratio between semiaxes are of a nonmonotonous character. The anticrosing effects are observed at the dependences of electron energy spectrum on the transversal size of the core-shell nanowire.
\end{abstract}

Key words: elliptic quantum wire, elliptic nanotube, energy spectrum

PACS: 73.21.Fg, 73.21. $\mathrm{Hb}, 73.21 . \mathrm{La}$

\section{Introduction}

Recently semiconductor nanowires have attracted great attention due to their small diameter that leads to one-dimensional electron systems as well as due to the possibility of being used as the building blocks in nanoscale electronics and photonics. The advantages of semiconductor nanowires compared to carbon nanotubes lie in controllability of conduction types and in the formation of heterojunctions and superlattices. These lead to a broad range of applications such as single electron transistors, light emitting or laser diodes and chemical sensors. Such potential capabilities can be greatly enhanced by introducing heterostructures within semiconductor nanowires. Modern technologies permit to grow the nanowires of different shape and structure. Among them there are the cylindrical, hexagonal and elliptical nanowires [1,2], multishell heterostructure nanowires [3] and semiconductor nanotubes $[4,5]$.

In order to apply the multishell heterostructure nanowires into different optical of the components devices it is necessary to investigate the quasiparticles energy dependences on the shape and geometrical size of the components of multishell nanowires.

The theory of quasiparticles spectra in complicated cylindrical semiconductor heterostructures was developed much earlier than the growth technology of multishell heterostructure nanowires [6]. Within the framework of this theory it is impossible to describe the properties of one-dimensional nanoheterostructures with the elliptic cross-section. These nanostructures can be grown using selective-area metalorganic vapor phase epitaxy or under the action of one axis pressure. The particular interest to the elliptic nanowires is also caused by the fact that the size quantization problem for a carrier with anisotropic effective mass parameters in a cylindrical well (as it is shown in [7]) is to be equivalent to that of a carrier with some isotropic effective mass in an elliptic well.

For the infinitely deep elliptic potential well model which can be used for the carriers in semiconductor nanowires embedded into dielectric matrices, there exists an exact solution of Schrodinger equation based on the Mathieu functions [8]. The approximated solutions can be obtained for the case of potential elliptic well of a finite depth [8,9]. The comparison of the results of numerical calculations within finite-difference method on a $400 \times 400$ uniform rectangular grid [9] with the

*E-mail: theorphys@chnu.cv.ua 
method of separation of radial and angular elliptic variables [8] proves that the second method can be applied to the ratio between the ellipse semiaxes $a / b<3$.

The size quantization problem for a carrier in core-shell elliptic semiconductor nanowire or elliptic nanotube has not been solved yet. Therefore, the peculiarities of the electron energy spectrum in such nanostructures are investigated in this paper.

\section{Hamiltonian of the system. Solutions of Schrodinger equation}

\subsection{Single elliptic nanowire}

The elliptic quantum wire (EQW) GaAs ("0") embedded into the semiconductor or dielectric matrix ("1") is under study. The coordinate system is chosen in such a way that Oz axis is directed along the wire axis. Electron potential energy and effective mass in Cartesian coordinates have the form

$$
\begin{aligned}
& \mu(x, y)= \begin{cases}\mu_{0}, & x^{2} / a^{2}+y^{2} / b^{2} \leqslant 1, \\
\mu_{1}, & x^{2} / a^{2}+y^{2} / b^{2}>1,\end{cases} \\
& U(x, y)= \begin{cases}0, & x^{2} / a^{2}+y^{2} / b^{2} \leqslant 1, \\
V, & x^{2} / a^{2}+y^{2} / b^{2}>1,\end{cases}
\end{aligned}
$$

where $a$ and $b$ are the ellipse semiaxes.

Electron can perform a free movement in the direction along the quantum wire. Due to the tunnel effect, the wave function of quasiparticle can penetrate into the medium " 1 " and part of the energy caused by the longitudinal movement of electron is $E_{z}=\hbar^{2} k_{z}^{2} / 2 \mu^{*}$, where $\mu^{*}$ is averaged effective mass, equal to $\mu_{0}$ in the case of infinite potential barrier. The energy caused by the transversal movement of quasiparticle is found from the Schrodinger equation

$$
-\frac{\hbar^{2}}{2} \nabla \frac{1}{\mu(x, y)} \nabla \Psi(x, y)+U(x, y) \Psi(x, y)=E \Psi(x, y) .
$$

Setting (1), (2) into (3), the following equations are obtained for every medium

$$
\Delta \Psi^{(i)}(x, y)+k_{i}^{2} \Psi^{(i)}(x, y)=0,(i=0,1)
$$

where $k_{i}^{2}=2 \mu_{i} \hbar^{-2}\left(E-V_{i}\right), V_{0}=0, V_{1}=V$.

Taking into account the elliptic symmetry, equation (4) is convenient to be solved in elliptic coordinates $(\xi, \eta, z)$ bound to the Cartesian by the relationships

$$
\left.\begin{array}{l}
x=f \cosh \xi \cos \eta, \quad 0 \leqslant \xi<\infty \\
y=f \sinh \xi \sin \eta, \quad 0 \leqslant \eta<2 \pi \\
z=z, \quad-\infty<z<+\infty
\end{array}\right\}
$$

where $f=\sqrt{a^{2}-b^{2}}$ is the focus distance, $\xi$ is the radial coordinate and $\eta$ is the angular coordinate. The radial coordinate $(\xi)$ is defined by the ratio between ellipse semiwidths $(\tanh \xi=b / a)$. Transiting in equation (4) from Cartesian coordinates to the elliptic, one can get

$$
\left[\frac{\partial^{2}}{\partial \xi^{2}}+\frac{\partial^{2}}{\partial \eta^{2}}+\frac{f^{2} k_{i}^{2}}{2}(\cosh 2 \xi-\cos 2 \eta)\right] \Psi^{(i)}(\xi, \eta)=0, \quad(i=0,1) .
$$

The wave function permits the separation of the variables only for elliptic quantum well with impenetrable walls. Then, the quantum states of quasiparticle are characterized by the definite value of quantum number $m$

$$
\Psi_{m}(\xi, \eta)=R_{m}(\xi) \theta_{m}(\eta)
$$

Using the variable separation for elliptic quantum well of the finite depth with $a / b<3$ we can obtain the size quantization spectra of carriers with high accuracy. $R_{m}^{(i)}(\xi)$ is the radial and $\theta_{m}^{(i)}(\eta)$ is the angular part of electron wave functions in $i$ th medium, satisfying Mathieu equations

$$
\begin{aligned}
\partial^{2} \theta_{m}^{(i)}(\eta) / \partial \eta^{2}+\left(c-2 q_{i} \cos 2 \eta\right) \theta_{m}^{(i)}(\eta) & =0 \\
\partial^{2} R_{m}^{(i)}(\xi) / \partial \xi^{2}-\left(c-2 q_{i} \cosh 2 \xi\right) R_{m}^{(i)}(\xi) & =0
\end{aligned}
$$


where $q_{i}=f^{2} k_{i}^{2} / 4, c$ is the separating constant. The solution of radial equation (9) is the linear combination of even $(e)$ and odd $(o)$ modified Mathieu functions of first and second type

$$
\begin{aligned}
& R_{m}^{e}(q, \xi)=\left\{\begin{array}{cc}
A_{m}^{e} J e_{m}(q, \xi)+B_{m}^{e} N e_{m}(q, \xi), & q>0, \\
A_{m}^{e} I e_{m}(q, \xi)+B_{m}^{e} K e_{m}(q, \xi), & q<0,
\end{array}\right. \\
& R_{m}^{o}(q, \xi)=\left\{\begin{array}{cc}
A_{m}^{o} J o_{m}(q, \xi)+B_{m}^{o} N o_{m}(q, \xi), & q>0, \\
A_{m}^{o} I o_{m}(q, \xi)+B_{m}^{o} K o_{m}(q, \xi), & q<0,
\end{array}\right.
\end{aligned}
$$

where $A_{m}^{e}, A_{m}^{o}, B_{m}^{e}, B_{m}^{o}$ - the coefficients determined by fitting and normalizing conditions.

The energy spectrum of quasiparticle is obtained within the fitting conditions for the wave function. In case of impenetrable walls for the elliptic quantum wire, the even and odd wave functions at the surface of elliptic cylinder $\left(\xi=\xi_{0}\right)$ are equal to zero. Their radial parts would contain only the functions $J e_{m}(q, \xi)$ and $J_{o_{m}}(q, \xi)\left(B_{m}^{e}=B_{m}^{o}=0\right)$ in analogy to the cylindrical functions. Thus, the energies of even and odd states in EQW are characterized by the fixed magnitude of quantum number $m$ and are defined from the equations

$$
\left.J e_{m}(q, \xi)\right|_{\xi=\xi_{0}}=0, \quad m=0,1,2, \ldots ;\left.\quad J o_{m}(q, \xi)\right|_{\xi=\xi_{0}}=0, \quad m=1,2, \ldots
$$

The values $q_{n m}^{e(o)}=f^{2} E_{n m}^{e(o)} \mu_{0} / 2 \hbar^{2}$, satisfying equations (12), determine the quasiparticle discrete energy levels $E_{n m}^{e(o)}$, where $n=1,2, \ldots$ is quantum number fixed by the order of the root of respective equations $(12)$.

In the case of elliptic quantum well with finite depth, the wave functions satisfy the boundary conditions

$$
\begin{gathered}
\left.\frac{1}{\mu_{0}} \frac{\partial R_{m}^{e(0)}\left(q_{0}, \xi\right) / \partial \xi}{R_{m}^{e(0)}\left(q_{0}, \xi\right)}\right|_{\xi=\xi_{0}}=\left.\frac{1}{\mu_{1}} \frac{\partial R_{m}^{e(1)}\left(q_{1}, \xi\right) / \partial \xi}{R_{m}^{e(1)}\left(q_{1}, \xi\right)}\right|_{\xi=\xi_{0}}, \\
\left.\frac{1}{\mu_{0}} \frac{\partial R_{m}^{o(0)}\left(q_{0}, \xi\right) / \partial \xi}{R_{m}^{o(0)}\left(q_{0}, \xi\right)}\right|_{\xi=\xi_{0}}=\left.\frac{1}{\mu_{1}} \frac{\partial R_{m}^{o(1)}\left(q_{1}, \xi\right) / \partial \xi}{R_{m}^{o(1)}\left(q_{1}, \xi\right)}\right|_{\xi=\xi_{0}} .
\end{gathered}
$$

The finiteness conditions of the radial wave functions determine the coefficients $B_{m}^{e(0)}=$ $B_{m}^{o(0)}=0$ and $A_{m}^{e(1)}=A_{m}^{o(1)}=0$. Setting (10), (11) into (13), (14) the dispersion equations are obtained for electron spectrum in elliptic nanowire with finite potential barrier.

\subsection{Complicated elliptic quantum wire}

The elliptic quantum thread ("0") covered with the elliptic shell ("1") embedded into the dielectric matrix ("2") is under study. It is assumed that electron cannot penetrate into the matrix and its potential energy in Cartesian coordinates is as follows

$$
U(x, y)= \begin{cases}V_{0}, & x^{2} / a_{0}^{2}+y^{2} / b_{0}^{2} \leqslant 1 \\ V_{1}, & x^{2} / a_{0}^{2}+y^{2} / b_{0}^{2}>1 \cap x^{2} / a_{1}^{2}+y^{2} / b_{1}^{2} \leqslant 1, \\ \infty, & x^{2} / a_{1}^{2}+y^{2} / b_{1}^{2}>1,\end{cases}
$$

where $a_{0}, b_{0}$ and $a_{1}, b_{1}$ are the semiaxis of inner and outer ellipse, respectively.

The wave function at $\xi=\xi_{1}=\operatorname{arctanh}\left(b_{1} / a_{1}\right)$ is equal to zero and at the media interface $\xi=\xi_{0}=\operatorname{arctanh}\left(b_{0} / a_{0}\right)$ it satisfies the boundary condition (13), (14), which determines the dispersion equation for the electron energy spectrum. For the case $V_{1}>V_{0}$ there is obtained

$$
\begin{cases}\frac{J_{m}^{\prime}\left(q_{0}, \xi_{0}\right)}{\mu_{0} J_{m}\left(q_{0}, \xi_{0}\right)}-\frac{J_{m}^{\prime}\left(q_{1}, \xi_{0}\right) N_{m}\left(q_{1}, \xi_{1}\right)-N_{m}^{\prime}\left(q_{1}, \xi_{0}\right) J_{m}\left(q_{1}, \xi_{1}\right)}{\mu_{1}\left[J_{m}\left(q_{1}, \xi_{0}\right) N_{m}\left(q_{1}, \xi_{1}\right)-N_{m}\left(q_{1}, \xi_{0}\right) J_{m}\left(q_{1}, \xi_{1}\right)\right]}=0, & q_{1} \geqslant 0 \\ \frac{J_{m}^{\prime}\left(q_{0}, \xi_{0}\right)}{\mu_{0} J_{m}\left(q_{0}, \xi_{0}\right)}-\frac{I_{m}^{\prime}\left(q_{1}, \xi_{0}\right) K_{m}\left(q_{1}, \xi_{1}\right)-K_{m}^{\prime}\left(q_{1}, \xi_{0}\right) I_{m}\left(q_{1}, \xi_{1}\right)}{\mu_{1}\left[I_{m}\left(q_{1}, \xi_{0}\right) K_{m}\left(q_{1}, \xi_{1}\right)-K_{m}\left(q_{1}, \xi_{0}\right) I_{m}\left(q_{1}, \xi_{1}\right)\right]}=0, & q_{1}<0\end{cases}
$$


where $q_{i}=f^{2} k_{i}^{2} / 4, k_{i}^{2}=2 \mu_{i} \hbar^{-2}\left(E_{n m}^{e(o)}-V_{i}\right)$.

The numerical calculations performed over equations (16) make it possible to obtain even and odd electron energies $E_{n m}^{e(o)}, n=1,2,3, \ldots$ is quantum number fixing the order of the corresponding root of dispersion equation. Such solutions are possible only for the complicated elliptic quantum wires with interfaces between ellipses which have equal focus distances since this is demanded by the elliptic coordinate system (5).

\subsection{Elliptic semiconductor nanotube}

The elliptic semiconductor nanotube embedded into the dielectric or semiconductor matrix is under study. Potential energy of the quasiparticle in Cartesian coordinates is as follows

$$
U(x, y)=\left\{\begin{array}{lll}
V, & x^{2} / a_{0}^{2}+y^{2} / b_{0}^{2} \leqslant 1 & \cup x^{2} / a_{1}^{2}+y^{2} / b_{1}^{2}>1 \\
0, & x^{2} / a_{0}^{2}+y^{2} / b_{0}^{2}>1 & \cap x^{2} / a_{1}^{2}+y^{2} / b_{1}^{2} \leqslant 1
\end{array}\right.
$$

In the case of elliptic quantum well with infinite depth $(V=\infty)$ the even and odd radial wave functions (10), (11) are equal to zero at the interfaces $\xi=\xi_{0}$ and $\xi=\xi_{1}$. These conditions make it possible to obtaine the exact electron energy spectrum in elliptic nanotube with arbitrary ratio between semiaxes of inner and outer ellipses but with equal focuses.

In the case of the elliptic quantum well with finite depth, using the conditions (13), (14) of wave function and density of probability current continuity for the even and odd states at the both interfaces there is obtained the electron energy spectrum in nanowires embedded into a semiconductor matrix.

\section{Results and discussion}

\subsection{Elliptic nanowire GaAs}

The computer calculations over equations (12) were performed using the value of the electron effective mass in the bulk crystal GaAs: $\mu_{0}=0.067 m_{0}$, where $m_{0}$ is the pure electron mass.

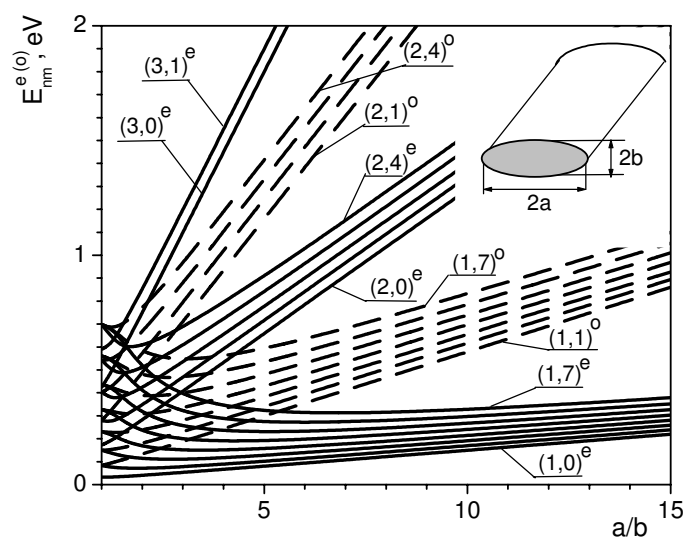

Figure 1. Dependence of electron energy spectrum in elliptic quantum wires GaAs with impenetrable walls on the ratio between semiaxes. Solid curves - even states, dash curves - odd states.

Figure 1 presents the results obtained for the energies of an electron transversal movement in elliptic nanowire GaAs with impenetrable walls depending on the ratio between ellipse semiaxes at its constant square $S=\pi a b$, corresponding to the square of the circle with radius $R=10 \mathrm{~nm}$. The even states are shown by solid curves and the odd ones by dashed. Figure 1 proves that even and odd states create the sets of the levels. Herein the energies of even states are located lower than 


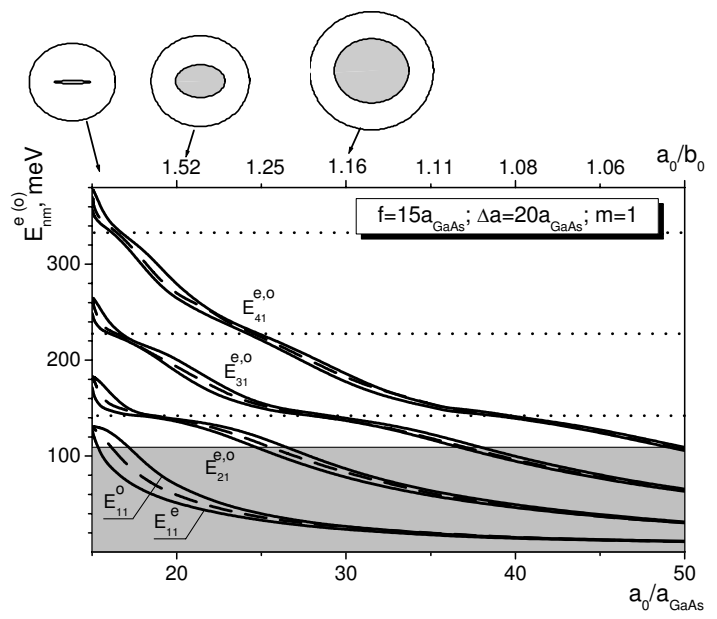

Figure 2. Dependences of even $\left(E_{n m}^{e}\right)$ and odd $\left(E_{n m}^{o}\right)$ energy states of electron in EQW at $m=1$ (solid curves) and energies $\left(E_{n m}\right)$ in the respective CQW (dashed curves) on $a_{0}$.

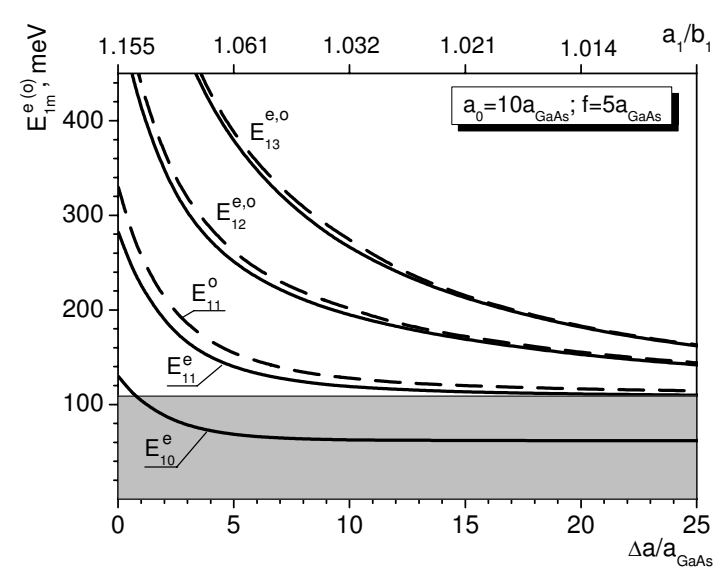

Figure 3. Dependences of even (solid curves) and odd (dashed curves) states of electron energies in core-shell elliptic quantum well at $\mathrm{n}=1$ on the thickness of elliptic shell.

the respective energies of odd states. At the ellipse semiaxes ratio $a / b=1$, the electron energy spectrum is degenerated. The ground electron state (the even one) is non-degenerated just like in cylindrical nanowire.

\subsection{Elliptic core-shell nanowire $\mathrm{GaAs} / \mathrm{Al}_{x} \mathrm{Ga}_{1-x} \mathrm{As} / \mathrm{dielectric}$}

In figure 2 the electron energies of even and odd states (solid curves) with $k_{z}=0, m=1$ are presented for the complicated elliptic quantum wire $\mathrm{GaAs} / \mathrm{Al}_{\mathrm{x}} \mathrm{Ga}_{1-\mathrm{x}} \mathrm{As}$ as functions of the magnitude of semiaxis $a_{0}$ of the inner thread (GaAs) of quantum wire at the constant thickness of the shell $\left(\mathrm{Al}_{\mathrm{x}} \mathrm{Ga}_{1-\mathrm{x}} \mathrm{As}\right)$ in the direction of OX axis $\left(\Delta a=20 a_{\mathrm{GaAs}}\right)$ and constant focus distance $\left(f=15 a_{\mathrm{GaAs}}\right)$ of both confining elliptic cylinders $\left(a_{\mathrm{GaAs}}=5,65 \AA\right.$ is the lattice constant of bulk crystal GaAs). The analogous dependences for the similar complicated cylindrical quantum wire (CQW) (dash curves) are shown for comparison. Herein, the radii $r_{0}$ and $r_{1}$ for CQW were determined by the condition of equality of cross-section square of the inner thread and shell for the elliptic and cylindrical quantum wire: $r_{0}=\sqrt{a_{0} b_{0}}, r_{1}=\sqrt{a_{1} b_{1}}$.

From figure 2 it is clear that the energies of electron even states in EQW are always smaller while those of the odd ones are bigger than the corresponding energies in CQW. Such a splitting is big for the lower energy levels and decreases for the states with bigger main quantum number. This is explained by the fact that the ratio $a_{0} / b_{0}$ is always bigger than $a_{1} / b_{1}$ (since the focus distance of both ellipses are equal). Since the skip of potential energy at the inner interface is $\mathrm{V}=109 \mathrm{meV}$, then the electron in the states with bigger energy "feels" this interface less. At the increase of $a_{0}$, the EQW elliptic cross-sections approach the circle (this is obvious from $\mathrm{a}_{0} / \mathrm{b}_{0}$ magnitudes shown at the upper horizontal axis of the figure and the pictures of the quantum wire cross-sections), the energies of even and odd electron states coincide and tend to the respective values in CQW. Toned region in figure 2 corresponds to the energies lower than the potential barrier. Here, the energy spectrum is monotonously decreasing. As for the energies higher than the potential barrier, one can see the anticrossing effect because the nanosystem under research consists of two potential wells, each of which has its own system of energy levels, the crossings between which are forbidden for the states with the same quantum number $m$. Consequently, the dependence of electron energy spectrum on $a_{0}$ magnitude in the region higher than the potential barrier is of a nonmonotonous character. In the vicinity of the energy level anticrossings, the quasiparticle changes its location in nanosystem. 
Figure 3 shows the energy dependences of even $E_{1 m}^{e}$ (solid curves) and odd $E_{1 m}^{o}$ (dashed curves) of electron states on the thickness $(\Delta a)$ for the elliptic shell $\left(\mathrm{Al}_{\mathrm{x}} \mathrm{Ga}_{1-\mathrm{x}} \mathrm{As}\right)$ at $a_{0}=10 a_{\mathrm{GaAs}}$ and $f=5 a_{\mathrm{GaAs}}$. Figure proves that these energies are monotonously decreasing. In the limit case $(\Delta a=0)$ there is obtained the EQW with impenetrable potential wells. When the thickness of the shell $(\Delta a)$ increases, the size quantization becomes weaker and potential energy of electron in the shell is finite, since the electron in the lowest energy state is bound by the potential well of the central part of the wire. At $\Delta a>8 a_{\mathrm{GaAs}}$, the energy dependence of the ground electron state on the thickness of the shell is saturating. The latter corresponds to the energy of electron in elliptic wire, embedded into the massive media $\mathrm{Al}_{\mathrm{x}} \mathrm{Ga}_{1-\mathrm{x}} \mathrm{As}$, which may be obtained from roots of equations (6), (7). The energies of the excited electron states becoming closer to each other, tend to the bottom of the potential well created by the elliptic shell. The magnitude of the splitting of even and odd energy states becomes smaller with the increase of the shell thickness because the ellipticity of the outer interface is decreasing (magnitude of the ratio between semiaxes $\left(a_{1} / b_{1}\right)$ is shown at the upper axis of the graphics).

\subsection{Elliptic nanotube $\ln P / \ln A s / \ln P$}

The computer calculations were performed using the following parameters of semiconductor bulk crystals: $\mu_{0}=0,0795 m_{0}, \mu_{1}=0,026 m_{0}$, are electron effective masses in InP and InAs, respectively, $V=0,33 \mathrm{eV}$ in the height of the potential barrier for the electron at the interface $\mathrm{InP} / \mathrm{InAs}, a_{\mathrm{InP}}=5,87 \AA, a_{\mathrm{InAs}}=6,06 \AA$ are the lattice constants.

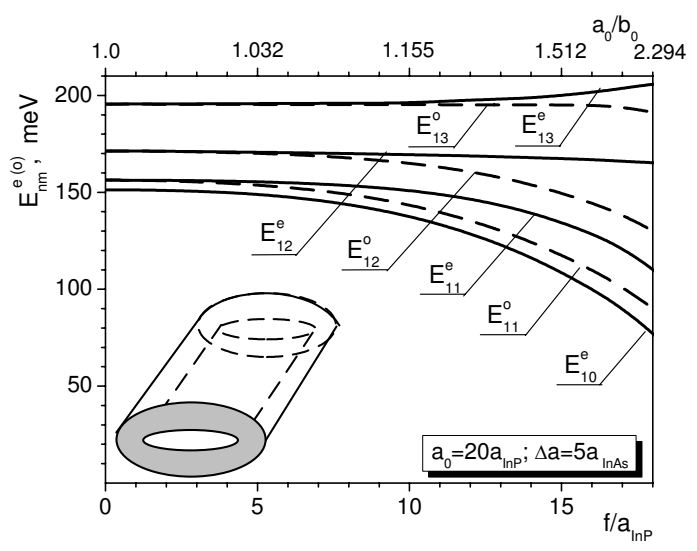

Figure 4. Dependences of even $E_{n m}^{e}$ (solid curves) and odd $E_{n m}^{o}$ (dashed curves) states of electron energies on the focus distance $f$ at the constant magnitudes $a_{0}=20 a_{\mathrm{InP}}, \Delta A=5 a_{\mathrm{InAs}}$.

Figure 4 presents the dependence of electron energy spectrum in nanotube on $f$ at $a_{0}=20 a_{\mathrm{InP}}$ and $\Delta a=5 a_{\text {InAs }}$ (values of the semiaxes ratio $a_{0} / b_{0}$ are noted on the top of figure). In the figure one can see that at $f=0$ (nanotube with circle cross-section) the energies of even and odd states coincide. The increase of focus distance causes the splitting of the energies of even and odd states due to the increase of the anisotropy of inner and outer elliptic interfaces.

\section{Conclusions}

In the elliptic coordinate system based on the Mathieu functions within the framework of the effective mass approximation, the energy spectra of electron are calculated: for the bare quantum wire GaAs, nanowire GaAs covered with the shell $\mathrm{Al}_{\mathrm{x}} \mathrm{Ga}_{1-\mathrm{x}}$ As with impenetrable walls for the quasiparticle and elliptic nanotube $\mathrm{InP} / \mathrm{InAs} / \mathrm{InP}$. The electron energy spectrum consists of a series of energy levels corresponding to the even and odd electron states. The results of investigation of electron energy spectrum in core-shell elliptic quantum wire and elliptic nanotube prove that the splitting between the energies of even and odd states depends on the ellipticity of both media 
interfaces. Herein, even a small deformation of cylindrical quantum wire or nanotube essentially effects the electron energy spectrum.

\title{
Acknowledgements
}

This work was financially supported by MPZ PP "Kolos".

\section{References}

1. Hu J., Bando Y., Liu Z., Sekiguchi T., Golberg D., Zhan J., J. Am. Chem. Society, 2003, 125, 11306.

2. Noborisaka J., Motohisa J., Fukui T., Appl. Phys. L., 2005, 86, 213102.

3. Noborisaka J., Motohisa J., Hara S., Fukui T., Appl. Phys. L., 2005, 87, 093109.

4. Mohan P., Motohisa J., Fukui T., Appl. Phys. L., 2006, 88, 133105.

5. Mohan P., Motohisa J., Fukui T., Appl. Phys. L., 2006, 88, 013110.

6. Tkach M., Quasiparticles in nanoheterosystems. Quantum dots and wires, Ruta, Chernivtsi, 2003, 312p.

7. Bejenari I., Kantser V., Myronov M., Mironov O., Leadley D., Semicond. Sci. Technol., 2004,19, 106.

8. Broek M., Peeters F., Physica E,2001, 11, 345.

9. Lew Yan Voon L., Galeriu C., Willatzen M., Physica E, 2003, 18, 547.

\section{Спектри електрона у складному еліптичному квантовому дроті}

\author{
В.А.Головацький, В.І.Гуцул \\ Чернівецький національний університет ім. Юрія Федьковича вул. Коцюбинського 2, 58012, Чернівці \\ Отримано 4 вересня 2006 р., в остаточному вигляді - 4 січня 2007 р. \\ В рамках наближення ефективних мас досліджено енергетичний спектр електрона в складному елі- \\ птичному квантовому дроті та напівпровідниковій еліптичній нанотрубці. В еліптичних координатах \\ на основі функцій Мат'є отримано розв'язок рівняння Шредінгера. Розраховано залежності спектру \\ розмірного квантування електрона від геометричних розмірів та форми складного квантового дроту \\ та нанотрубки. Показано, що еліптичність квантового дроту приводить до розщеплення енергети- \\ чних рівнів. Залежність парних та непарних електронних енергетичних станів від співвідношення \\ півосей еліпса має немонотонний характер. На залежності енергетичного спектру електрона від \\ поперечних розмірів складного квантового дроту спостерігається ефект розштовхування рівнів.
}

Ключові слова: еліптичний квантовий дріт, еліптична нанотрубка, енергетичний спектр

PACS: $73.21 . \mathrm{Fg}, 73.21 . \mathrm{Hb}, 73.21 . \mathrm{La}$ 
